\title{
Genetic characterisation of African swine fever viruses from outbreaks in southern Africa (1973-1999)
}

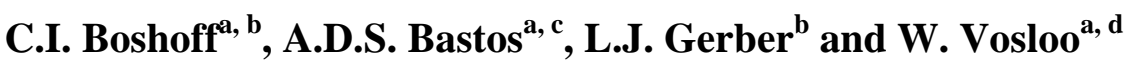 \\ ${ }^{\mathrm{a}}$ ARC-OVI, Exotic Diseases Division, Private Bag X05, Onderstepoort 0110, South \\ Africa \\ ${ }^{b}$ Department of Biomedical Sciences, Tshwane University of Technology, Pretoria, South \\ Africa \\ ${ }^{\mathrm{c}}$ Mammal Research Institute, Department of Zoology and Entomology, University of \\ Pretoria, Pretoria 0002, South Africa \\ ${ }^{\mathrm{d}}$ Department of Veterinary Tropical Diseases, University of Pretoria, Onderstepoort, \\ South Africa
}

\section{Abstract}

African swine fever (ASF) is a highly lethal and economically significant disease of domestic pigs in the southern African sub-region, where outbreaks regularly occur. There is anecdotal evidence suggesting that trans-boundary movement of infected animals may have played a role in precipitating widespread outbreaks in the past, however, since the 1970s outbreaks have generally been more localised, particularly in those countries where control of animal movement is strictly regulated. The origin and relatedness of regional ASF outbreaks was investigated here by means of a two-step genetic characterisation approach whereby $p 72$ gene sequencing was used to delineate genotypes, prior to intragenotypic resolution of viral relationships by central variable region (CVR) characterisation of the $9 R L$ ORF. In this manner, regional virus heterogeneity and epidemiological links between outbreaks could be assessed for the first time through phylogenetic analysis of the C-terminal end of the $p 72$ gene of viruses recovered from domestic pig outbreaks in southern Africa between 1973 and 1999. The phylogeny revealed the presence of 14 distinct $p 72$ genotypes of which 6 (genotypes XVII-XXII) 
were considered novel. Eight of these were country-specific with the remaining six having a trans-boundary distribution. CVR products were heterogeneous in size ranging from 377 bp to 533 bp across the 14 southern African genotypes. Within-genotype CVR comparisons revealed the presence of a genotype XIX virus with an extended field presence in South Africa (1985-1996) and permitted discrimination between three genotype VII viruses that were identical across the $p 72$ gene.

\section{Article Outline}

1. Introduction

2. Materials and methods

2.1. Virus isolates used in this study

2.2. Genomic amplification and nucleotide sequence determination

2.3. Sequence analysis

3. Results

3.1. $p 72$ gene phylogeny

3.2. Intra-genotypic resolution of homogeneous $p 72$ southern African genotypes

4. Discussion

Acknowledgements

References

\section{Introduction}

African swine fever (ASF) is an important disease of domestic pigs, and is caused by an icosahedral double stranded DNA virus that is presently the sole member of the Asfivirus genus within the family Asfarviridae (Dixon et al., 2000). The virus occurs naturally in both vertebrate and invertebrate sylvatic hosts throughout sub-Saharan Africa and is transmitted to domestic pigs when infected soft-shelled, eyeless ticks of the Ornithodoros moubata complex feed on them (reviewed by Penrith et al., 2004). A domestic pig cycle, which is apparently not reliant on the presence of the tick vector, is believed to occur in both West and East Africa (reviewed by Penrith et al., 2004) and is characterised by the 
presence of viruses that are genetically homogeneous across the $\mathrm{C}$-terminal end of the p72 gene (Bastos et al., 2003 and Lubisi et al., 2005).

ASF, initially termed 'East African swine fever' was first described from Kenya in 1921 (Montgomery, 1921), with the earliest record of the disease in South Africa dating back to 1926 (Steyn, 1932) from the northern area of this country (former Transvaal province) where contact between warthogs (Phacochoerus africanus) and domestic pigs was known to occur. Two outbreaks occurred in the Cape in 1933 ostensibly due to a consignment of infected pigs from the former Transvaal, which were followed by outbreaks until 1939 (Pini and Hurter, 1975). From 1939 onwards, all ASF outbreaks were restricted to the Transvaal (now Gauteng, Limpopo and Mpumulanga provinces) and demonstrated an apparent cyclical trend with numerous outbreaks being recorded between 1935 and 1939, 1951 and 1962, and between 1973 and 1975 (Pini and Hurter, 1975) and no reports for the intervening time periods. Of these, trans-boundary movement of infected pigs from Namibia to South Africa is believed to have precipitated the 1951 outbreaks, which were widespread, with later outbreaks being attributed to warthog presence.

The inability of ASF virus to induce neutralizing antibodies has hampered the prevention and control of the disease by vaccination. In the absence of effective vaccines, control of ASF in high-risk areas of Africa relies on the prevention of contact between domestic pigs and free-living reservoirs (Penrith et al., 2004) and on a slaughter-out policy in the event of an outbreak, which results in major economic losses. In southern Africa clinical disease is regularly recorded in domestic pigs, and is believed to result primarily from contact between domestic pigs and argasid ticks. However, as experimental evidence suggests that pigs can exceptionally become infected from eating infected warthog tissue (Thomson et al., 1980), and that virus transmission from bushpigs (Potamochoerus porcus) to domestic pigs is possible (Anderson et al., 1998), strict controls are imposed on contact between domestic pigs and all wildlife reservoirs, including ticks, wild suids and their meat products. This approach appears to be effective as outbreaks tend to be sporadic and locally restricted in those southern African countries that apply strict zoosanitary measures. However, exceptions to this primary tick-mediated transmission to pigs exist in areas such as the northern Tete province of Mozambique that borders Malawi. Control of ASF in areas like this, where apparently healthy, serologically 
positive domestic pigs occur in the absence of ticks, is more complex and requires community involvement and improved husbandry in order to be successful (Penrith, 2005 and Penrith et al., in press).

The formulation of appropriate disease control strategies requires intensive epidemiological investigations that benefit greatly from insights provided from retrospective analysis of the source and spread of historical outbreaks, as was the case for foot-and-mouth disease in southern Africa (Vosloo et al., 2006). Achieving this requires the availability of historical isolates and techniques capable of differentiating between strains. For ASF, studies of this nature have been impeded by the lack of discernible serotypes and could previously only be achieved by costly and time-consuming genomic characterisation methods such as restriction fragment length polymorphism (RFLP) analysis (Wesley and Tuthill, 1984, Dixon and Wilkinson, 1988 and Blasco et al., 1989). The availability of a PCR-sequencing method that permits rapid identification of the major genotypes (Bastos et al., 2003) and that has successfully been applied at a regional (Lubisi et al., 2005) and country level (Bastos et al., 2004), makes it possible to investigate the field heterogeneity of viruses causing recent and historical outbreaks of ASF in southern Africa for the first time. When used in conjunction with genetic characterisation of the central variable region (CVR) of the $9 R L$ open reading frame (ORF; Irusta et al., 1996), it is possible to resolve intra-genotypic relationships and in so doing provide new epidemiological insights in countries where the disease has previously been under-studied (Bastos et al., 2004 and Penrith et al., in press). A combined $p 72-$ CVR approach was therefore selected in this study that aims to determine the number of p72 genotypes involved in pig outbreaks in southern Africa between 1973 and 1999, and to investigate the relatedness of outbreaks caused by viruses of the same genotype.

\section{Materials and methods}

\subsection{Virus isolates used in this study}

For the purposes of this study, southern African countries are defined as those predominantly situated south of latitude $15^{\circ} 00^{\prime} \mathrm{S}$ and include South Africa, Namibia, Mozambique, Botswana and Zimbabwe. Twenty and 23 cell-culture isolates derived from outbreaks in domestic swine in southern Africa (1973-1999) were selected for the $p 72$ 
sequencing and CVR characterisation components of this study, respectively (Table 1). Three-day-old cultures of primary pig bone marrow cells grown in Earles medium supplemented with $10 \%$ pig serum were used to isolate virus from spleen or lymph nodes of pigs showing clinical signs of ASF (Malmquist and Hay, 1960). Additional cell culture isolates were obtained from the World Reference Laboratory, Pirbright (Table 1). 
Table 1.

Summary of the ASF viruses and genes characterised in this study

\begin{tabular}{|c|c|c|c|c|c|c|c|c|}
\hline Virus name & $\begin{array}{l}\text { Geographical } \\
\text { origin }\end{array}$ & $\begin{array}{l}\text { Sampling } \\
\text { year }\end{array}$ & $\begin{array}{l}\text { p72 Genbank } \\
\text { accession no. }\end{array}$ & Reference & $\begin{array}{l}\text { p72 } \\
\text { genotype }\end{array}$ & $\begin{array}{l}\text { CVR } \\
\text { Genbank } \\
\text { accession no. }\end{array}$ & Reference & $\begin{array}{l}\text { CVR } \\
\text { length in } \\
\text { amino } \\
\text { acids }\end{array}$ \\
\hline $\mathrm{MOZ} / 1960^{\mathrm{a}}$ & $\begin{array}{l}\text { Tete province, } \\
\text { Mozambique }\end{array}$ & 1960 & AF270708 & $\begin{array}{l}\text { Bastos et } \\
\text { al. (2004) }\end{array}$ & V & AY274465 & $\begin{array}{l}\text { Bastos et } \\
\text { al. (2004) }\end{array}$ & 88 \\
\hline $\mathrm{ANG} / 70^{\mathbf{a}}$ & Angola & 1970 & AF301542 & $\begin{array}{l}\text { Bastos et } \\
\text { al. (2003) }\end{array}$ & I & - & - & - \\
\hline Lillie & South Africa & 1973 & DQ250109 & This study & $\mathrm{XX}$ & DQ250086 & This study & 52 \\
\hline 24823 & $\begin{array}{l}\text { Pietersburg, } \\
\text { South Africa }\end{array}$ & 1975 & DQ250110 & This study & XX & DQ250087 & This study & 52 \\
\hline MOZ/1979 & Mozambique & 1979 & AF270709 & $\begin{array}{l}\text { Bastos et } \\
\text { al. (2004) }\end{array}$ & V & AY274470 & $\begin{array}{l}\text { Bastos et } \\
\text { al. (2004) }\end{array}$ & 48 \\
\hline $\mathrm{NAM} / 1 / 80^{\mathbf{b}}$ & Namibia & 1980 & AF504881 & $\begin{array}{l}\text { Bastos et } \\
\text { al. (2003) }\end{array}$ & I & - & - & - \\
\hline $\mathrm{KAB} / 6 / 2 b^{\mathrm{a}, \mathbf{c}}$ & Zambia & 1983 & AY351522 & $\begin{array}{l}\text { Lubisi et } \\
\text { al. (2005) }\end{array}$ & XI & - & - & - \\
\hline $\mathrm{SUM} / 14 / 11^{\mathrm{a}, \mathrm{c}}$ & Zambia & 1983 & AY351542 & $\begin{array}{l}\text { Lubisi et } \\
\text { al. (2005) }\end{array}$ & XIII & - & - & - \\
\hline SPEC/53 & $\begin{array}{l}\text { Letaba, South } \\
\text { Africa }\end{array}$ & 1985 & DQ250111 & This study & XXI & DQ250088 & This study & 40 \\
\hline $\mathrm{NYA} / 1 / 2^{\mathrm{a}, \mathrm{c}}$ & Zambia & 1986 & AY351555 & Lubisi et & XIV & - & - & - \\
\hline
\end{tabular}




\begin{tabular}{|c|c|c|c|c|c|c|c|c|}
\hline Virus name & $\begin{array}{l}\text { Geographical } \\
\text { origin }\end{array}$ & $\begin{array}{l}\text { Sampling } \\
\text { year }\end{array}$ & $\begin{array}{l}\text { p72 Genbank } \\
\text { accession no. }\end{array}$ & Reference & $\begin{array}{l}\text { p72 } \\
\text { genotype }\end{array}$ & $\begin{array}{l}\text { CVR } \\
\text { Genbank } \\
\text { accession no. }\end{array}$ & Reference & $\begin{array}{l}\text { CVR } \\
\text { length in } \\
\text { amino } \\
\text { acids }\end{array}$ \\
\hline & & & & al. (2005) & & & & \\
\hline $\mathrm{SPEC} / 120$ & $\begin{array}{l}\text { Potgietersrus, } \\
\text { South Africa }\end{array}$ & 1987 & AF302812 & This study & XIX & DQ250089 & This study & 52 \\
\hline SPEC/125 & $\begin{array}{l}\text { Ellisras, South } \\
\text { Africa }\end{array}$ & 1987 & DQ250112 & This study & XIX & DQ250090 & This study & 52 \\
\hline SPEC/154 & Botswana & 1987 & DQ250113 & This study & VII & DQ250091 & This study & 60 \\
\hline SPEC/205 & $\begin{array}{l}\text { Windhoek, } \\
\text { Namibia }\end{array}$ & 1989 & DQ250114 & This study & I & DQ250092 & This study & 44 \\
\hline SPEC/207 & $\begin{array}{l}\text { Windhoek, } \\
\text { Namibia }\end{array}$ & 1989 & DQ250115 & This study & I & DQ250093 & This study & 44 \\
\hline SPEC/209 & $\begin{array}{l}\text { Windhoek, } \\
\text { Namibia }\end{array}$ & 1989 & DQ250116 & This study & I & DQ250094 & This study & 44 \\
\hline SPEC/245 & $\begin{array}{l}\text { Louis Trichardt, } \\
\text { South Africa }\end{array}$ & 1992 & DQ250117 & This study & XXII & DQ250095 & This study & 52 \\
\hline $\mathrm{SPEC} / 251$ & $\begin{array}{l}\text { Rustenburg, } \\
\text { South Africa }\end{array}$ & 1992 & DQ250118 & This study & XIX & DQ250096 & This study & 52 \\
\hline $\mathrm{ZIM} / 92 / 1^{\mathrm{a}}$ & $\begin{array}{l}\text { Gweru } \\
\text { Midlands, } \\
\text { Zimbabwe }\end{array}$ & 1992 & DQ250119 & This study & XVII & DQ250097 & This study & 48 \\
\hline $\mathrm{MZI} / 92 / 1^{\mathrm{a}}$ & Malawi & 1992 & AY351543 & Lubisi et & XII & - & - & - \\
\hline
\end{tabular}




\begin{tabular}{|c|c|c|c|c|c|c|c|c|}
\hline Virus name & $\begin{array}{l}\text { Geographical } \\
\text { origin }\end{array}$ & $\begin{array}{l}\text { Sampling } \\
\text { year }\end{array}$ & $\begin{array}{l}\text { p72 Genbank } \\
\text { accession no. }\end{array}$ & Reference & $\begin{array}{l}\text { p72 } \\
\text { genotype }\end{array}$ & $\begin{array}{l}\text { CVR } \\
\text { Genbank } \\
\text { accession no. }\end{array}$ & Reference & $\begin{array}{l}\text { CVR } \\
\text { length in } \\
\text { amino } \\
\text { acids }\end{array}$ \\
\hline & & & & al. (2005) & & & & \\
\hline SPEC/257 & $\begin{array}{l}\text { Ellisras, South } \\
\text { Africa }\end{array}$ & 1993 & DQ250120 & This study & III & DQ250098 & This study & 52 \\
\hline $\mathrm{SPEC} / 260$ & $\begin{array}{l}\text { Thabazimbi, } \\
\text { South Africa }\end{array}$ & 1993 & DQ250121 & This study & VII & DQ250099 & This study & 60 \\
\hline $\mathrm{SPEC} / 265$ & $\begin{array}{l}\text { Maputo, } \\
\text { Mozambique }\end{array}$ & 1994 & AF270710 & $\begin{array}{l}\text { Bastos et } \\
\text { al. (2003) }\end{array}$ & VI & AY274467 & $\begin{array}{l}\text { Bastos et } \\
\text { al. (2004) }\end{array}$ & 64 \\
\hline $\mathrm{MOZ} / 94 / 1^{\mathrm{a}}$ & $\begin{array}{l}\text { Maputo, } \\
\text { Mozambique }\end{array}$ & 1994 & AF270711 & $\begin{array}{l}\text { Bastos et } \\
\text { al. (2003) }\end{array}$ & VI & AY274468 & $\begin{array}{l}\text { Bastos et } \\
\text { al. (2004) }\end{array}$ & 64 \\
\hline $\mathrm{MOZ} / 94 / 8^{\mathrm{a}}$ & $\begin{array}{l}\text { Manica, } \\
\text { Mozambique }\end{array}$ & 1994 & AF270712 & $\begin{array}{l}\text { Bastos et } \\
\text { al. (2004) }\end{array}$ & VI & AY274469 & $\begin{array}{l}\text { Bastos et } \\
\text { al. (2004) }\end{array}$ & 64 \\
\hline $\mathrm{UGA} / 1 / 95^{\mathrm{a}}$ & Uganda & 1995 & AF449475 & $\begin{array}{l}\text { Bastos et } \\
\text { al. (2003) }\end{array}$ & IX & - & - & - \\
\hline $\mathrm{UGA} / 3 / 95^{\mathrm{a}}$ & Uganda & 1995 & AF449476 & $\begin{array}{l}\text { Bastos et } \\
\text { al. (2003) }\end{array}$ & $X$ & - & - & - \\
\hline NAM/1/95 & $\begin{array}{l}\text { Windhoek, } \\
\text { Namibia }\end{array}$ & 1995 & DQ250122 & This study & XVIII & DQ250100 & This study & 84 \\
\hline $\mathrm{RSA} / 1 / 95$ & $\begin{array}{l}\text { Hoedspruit, } \\
\text { South Africa }\end{array}$ & 1995 & DQ250123 & This study & $\mathrm{XX}$ & DQ250101 & This study & 80 \\
\hline $\mathrm{RSA} / 5 / 95$ & Ellisras, South & 1995 & DQ250124 & This study & III & DQ250102 & This study & 52 \\
\hline
\end{tabular}




\begin{tabular}{|c|c|c|c|c|c|c|c|c|}
\hline Virus name & $\begin{array}{l}\text { Geographical } \\
\text { origin }\end{array}$ & $\begin{array}{l}\text { Sampling } \\
\text { year }\end{array}$ & $\begin{array}{l}\text { p72 Genbank } \\
\text { accession no. }\end{array}$ & Reference & $\begin{array}{l}\text { p72 } \\
\text { genotype }\end{array}$ & $\begin{array}{l}\text { CVR } \\
\text { Genbank } \\
\text { accession no. }\end{array}$ & Reference & $\begin{array}{l}\text { CVR } \\
\text { length in } \\
\text { amino } \\
\text { acids }\end{array}$ \\
\hline & Africa & & & & & & & \\
\hline RSA/1/96 & $\begin{array}{l}\text { Gravelotte, } \\
\text { South Africa }\end{array}$ & 1996 & DQ250125 & This study & XXI & DQ250103 & This study & 40 \\
\hline $\mathrm{RSA} / 2 / 96$ & $\begin{array}{l}\text { Pienaarsrivier, } \\
\text { South Africa }\end{array}$ & 1996 & DQ250126 & This study & XIX & DQ250104 & This study & 52 \\
\hline $\mathrm{RSA} / 3 / 96$ & $\begin{array}{l}\text { Pienaarsrivier, } \\
\text { South Africa }\end{array}$ & 1996 & DQ250127 & This study & XIX & DQ250105 & This study & 52 \\
\hline MAD/1/98 & Madagascar & 1998 & AF270706 & $\begin{array}{l}\text { Bastos et } \\
\text { al. (2003) }\end{array}$ & II & AY274471 & $\begin{array}{l}\text { Bastos et } \\
\text { al. (2004) }\end{array}$ & 40 \\
\hline MOZ/A-98 & $\begin{array}{l}\text { Tete, } \\
\text { Mozambique }\end{array}$ & 1998 & AY274452 & $\begin{array}{l}\text { Bastos et } \\
\text { al. (2004) }\end{array}$ & VIII & AY274461 & $\begin{array}{l}\text { Bastos et } \\
\text { al. (2004) }\end{array}$ & 92 \\
\hline MOZ/60-98 & $\begin{array}{l}\text { Tete, } \\
\text { Mozambique }\end{array}$ & 1998 & AY274455 & $\begin{array}{l}\text { Bastos et } \\
\text { al. (2004) }\end{array}$ & II & AY274472 & $\begin{array}{l}\text { Bastos et } \\
\text { al. (2004) }\end{array}$ & 40 \\
\hline $\mathrm{RSA} / 1 / 98$ & $\begin{array}{l}\text { Potgietersrus, } \\
\text { South Africa }\end{array}$ & 1998 & AF302818 & $\begin{array}{l}\text { Bastos et } \\
\text { al. (2003) }\end{array}$ & VII & DQ250106 & This study & 56 \\
\hline BOT/1/99 & $\begin{array}{l}\text { Sherwood, } \\
\text { Botswana }\end{array}$ & 1999 & AF504886 & $\begin{array}{l}\text { Bastos et } \\
\text { al. (2003) }\end{array}$ & III & DQ250107 & This study & 56 \\
\hline $\mathrm{RSA} / 1 / 99 \mathrm{~W}^{\mathbf{b}}$ & South Africa & 1999 & AF302818 & $\begin{array}{l}\text { Bastos et } \\
\text { al. (2003) }\end{array}$ & IV & DQ250108 & This study & 52 \\
\hline TAN/1/01 & Tanzania & 2001 & AY494552 & Lubisi et & XV & - & - & - \\
\hline
\end{tabular}




\begin{tabular}{|l|l|l|l|l|l|l|l|}
\hline Virus name & $\begin{array}{l}\text { Geographical } \\
\text { origin }\end{array}$ & $\begin{array}{l}\text { Sampling } \\
\text { year }\end{array}$ & $\begin{array}{l}p 72 \text { Genbank } \\
\text { accession no. }\end{array}$ & Reference & $\begin{array}{l}\text { p72 } \\
\text { genotype }\end{array}$ & $\begin{array}{l}\text { CVR } \\
\text { Genbank } \\
\text { accession no. }\end{array}$ & $\begin{array}{l}\text { CVR } \\
\text { Reference } \\
\text { aming } \\
\text { acids }\end{array}$ \\
\hline & & & al. (2005) & & & \\
\hline ZAM/02/1 & Zambia & 2001 & AY494559 & $\begin{array}{l}\text { Lubisi et } \\
\text { al. (2005) }\end{array}$ & I & - & - \\
\hline TAN/2003/1 & Tanzania & 2003 & AY494550 & $\begin{array}{l}\text { Lubisi et } \\
\text { al. (2005) }\end{array}$ & XVI & - & - \\
\hline
\end{tabular}

${ }^{a}$ Viruses supplied by the Institute for Animal Health, Pirbright, UK.

${ }^{\mathrm{b}}$ Warthog Phacochoerus africanus origin.

${ }^{\mathrm{c}}$ Ornithodoros tick origin. 


\subsection{Genomic amplification and nucleotide sequence determination}

DNA was extracted from $200 \mu$ aliquots of cell culture specimens for use in two genomic amplification reactions, namely the C-terminal end of the $p 72$ gene and the CVR of the 9RL ORF. These gene regions were amplified using primers and cycling conditions described previously (Bastos et al., 2003 and Bastos et al., 2004). Bands of the expected size (478 bp for $p 72$, variable for CVR) were excised from the gel and purified by means of the Nucleospin extract purification system (Macherey-Nagel). The purified products were used as template for manual sequencing (Bastos et al., 2003), or for automated cycle sequencing with BigDye v3.0 (Applied Biosystems). Two independent amplification and sequencing reactions were performed per isolate with each of the PCR primers.

\subsection{Sequence analysis}

The $20 p 72$ gene sequences generated in this study were aligned with at least 1 virus representative of each of the $16(\mathrm{I}-\mathrm{XVI})$ known $p 72$ genotypes (Table 1) resulting in a final dataset comprising 42 taxa and 411 bp corresponding to nucleotide positions 14941905 of the Uganda strain (Yu et al., 1996). Neighbor-joining (NJ) and minimum evolution (ME) trees were constructed in MEGA3 (Kumar et al., 2004), with node reliability being assessed by 10,000 bootstrap replications. The best-fit model selected in ModelTest (Posada and Crandall, 1998) was used for maximum likelihood (ML) analyses performed in PAUP* version 4.0b10 (Swofford, 2003) and for Bayesian inference in MrBayes v3.04 (Huelsenbeck and Ronquist, 2003). ML and maximum parsimony (MP) trees were constructed using heuristic searches and tree bisection-reconnection (TBR) branch swapping, with nodal support being assessed by 100 and 400 nonparametric bootstrap replications, respectively. For Bayesian inference, four chains were run simultaneously using the default heating and swap parameters and the first 3000 trees were discarded as 'burn-in'.

\section{Results}

\section{1. p72 gene phylogeny}

The final $p 72$ dataset comprising 42 taxa displayed AT bias (57\%) with empirical base frequencies for A, C, G and T each being 0.2733, 0.2408, 0.1874 and 0.2986 . The 
$\mathrm{HKY}+I+\Gamma$ model was identified as the best-fit model in ModelTest with a transition:transversion ratio $(R)$ of 5.6248, gamma distribution shape parameter $(\Gamma)$ of 0.7455 and proportion of invariant sites $(I)$ of 0.6462 . Of the 411 nucleotide sites included in the analyses, 60 were variable and 37 were parsimony informative. Two equally parsimonious trees, 96 steps long and with $\mathrm{CI}=0.677, \mathrm{RI}=0.858$ and $\mathrm{RC}=0.581$ were recovered with equal weighting whilst character re-weighting with maximum RC indexes resulted in two equally parsimonious trees (59.38 steps long, $\mathrm{CI}=0.867, \mathrm{RI}=0.929$ and $\mathrm{RC}=0.805)$.

Trees with similar topology and bootstrap support values were recovered using NJ and $\mathrm{ME}$, but differences were noted when comparing these phenetic trees with those obtained with MP, ML and Bayesian inference. In particular, genotypes XIX and XX, which collapsed into a polytomy with the latter methods of analysis, had moderate levels of bootstrap support (61-72\%) with the distance methods (Fig. 1). This was probably due to low levels of between-genotype genetic distances obtained for viruses representative of genotypes XIX and XX, which did not meet the minimum between-genotype pairwise distance of $0.96 \%$ previously suggested as a guide for delineating genotypes in conjunction with the phylogeny (Bastos et al., 2003). Despite this, the 42 viruses clustered within 22 discrete lineages, representative of distinct genotypes. Of these, 16 coincided with genotypes identified by distance methods in previous studies (Bastos et al., 2003 and Lubisi et al., 2005), with six being novel and confined to the southern African region. The phylogeny further revealed that the 22 genotypes clustered within 3 discrete evolutionary lineages, labelled I-III (Fig. 1) corresponding with 3 geographically distinct localities. Lineage I comprised 13 genotypes from southern and West Africa, lineage II consisted of 7 genotypes primarily of East African origin (as defined by Lubisi et al., 2005), whilst lineage III was made up of two exclusively East African genotypes (IX and X). 
openUP (July 2007)

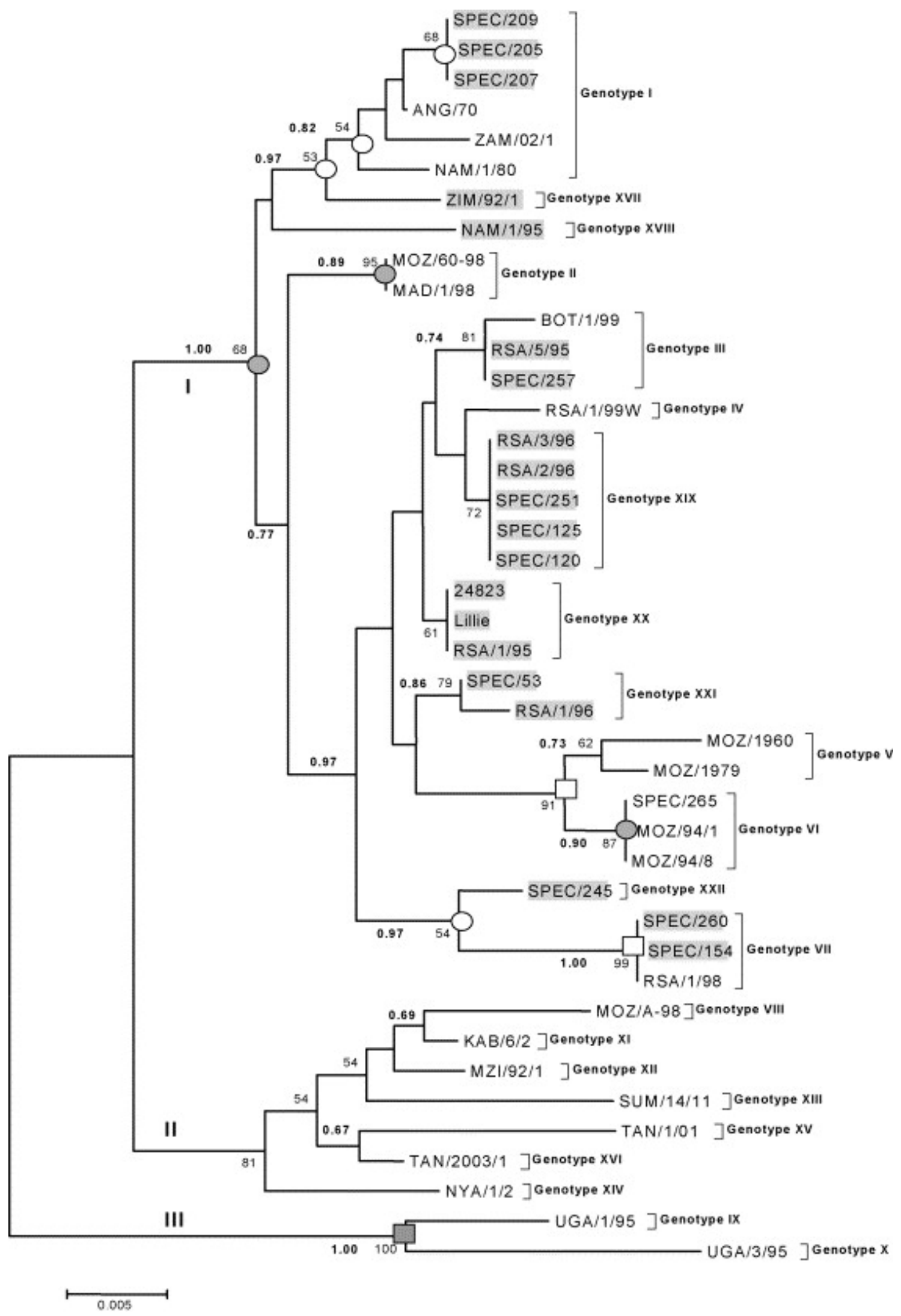


Fig. 1. Neighbor-joining tree depicting $p 72$ gene relationships of African swine fever viruses. Genotypes recovered from previous studies (I-X, Bastos et al., 2003 and IIXVI, Lubisi et al., 2005) follow the designations used in those studies, whilst the six new southern African genotypes recovered in this study are designated XVII-XXII. Bootstrap values $>50 \%$ obtained following 10,000 pseudoreplications are indicated next to each node together with the posterior probability value obtained from Bayesian inference. Nodes that had high levels of support with both parsimony and maximum likelihood are indicated as follows: open circles: $51-65 \%$, closed circles: $66-75 \%$, open squares: $85-$ 95\% and closed squares: $96-100 \%$. Viruses characterised in this study have a shaded background.

Of the 22 genotypes recovered, 14 were associated with domestic pig outbreaks in southern Africa and 8 appear to be country-specific (Table 2). However, at least four were shown to have a trans-boundary distribution, namely genotypes V and VIII which are shared between Mozambique and countries neighboring its northern borders, and genotypes III and VII which were recovered from northern South Africa and neighboring Botswana. The phylogeny also revealed links between southern African viruses that caused temporally unrelated outbreaks and was able to distinguish between viruses previously believed to have been part of a single epizootic. For example, five identical genotype XIX viruses were recovered from temporally unrelated outbreaks occurring in South Africa in 1987, 1992 and 1996. In contrast two 1995 South African outbreak strains (RSA/1/95 and RSA/5/95) were genetically unrelated, belonging to two different genotypes (genotypes XX and III, respectively). 
Table 2.

Summary of CVR size and sequence variation of African swine fever viruses of domestic pig origin representative of the 14 southern African $p 72$ genotypes, and their geographical distribution

\begin{tabular}{|c|c|c|c|c|c|}
\hline $\begin{array}{l}\text { p72 } \\
\text { genotype }\end{array}$ & $\begin{array}{l}\text { Southern African countries (other } \\
\text { African countries }^{\text {a }} \text { ) }\end{array}$ & $\begin{array}{l}\text { Field } \\
\text { presence }\end{array}$ & $\begin{array}{l}\text { No. of } \\
\text { viruses } \\
\text { (no. of } \\
\text { CVR } \\
\text { variants) }\end{array}$ & $\begin{array}{l}\text { Southern African CVR sequence } \\
\text { variants }\end{array}$ & $\begin{array}{l}\text { CVR-PCR } \\
\text { product size }^{\text {range }}\end{array}$ \\
\hline I & $\begin{array}{l}\text { Namibia (Angola, Benin, Cameroon, } \\
\text { Côte d'Ivoire, DRC, Gambia, Ghana, } \\
\text { Nigeria, Togo, Senegal, Zambia, } \\
\text { Zimbabwe) }\end{array}$ & 1989 & $3(1)$ & B N A F N B N A A A F & 389 \\
\hline II & Madagascar, Mozambique (Zambia) & 1998 & $2(1)$ & B N D B N D B N A A & 377 \\
\hline \multirow[t]{2}{*}{ III } & Botswana, South Africa & 1993-1999 & $3(2)$ & B V W V W V V N A A B A G & $413-425$ \\
\hline & & & & B V W V W V V N A A A B A G & \\
\hline IV & South Africa & 1999 & $1(1)$ & B V W V W V V N A A B A G & 413 \\
\hline \multirow[t]{2}{*}{$\mathrm{V}$} & Mozambique (Malawi) & 1960-1979 & $2(2)$ & $\begin{array}{l}\text { A B N A A A A A B N N B A B N B } \\
\text { A B M A A }\end{array}$ & $401-521$ \\
\hline & & & & A B M A B N B A B M A A & \\
\hline VI & Mozambique & 1994 & $3(1)$ & A A B A B N A B A B N B A B M A & 449 \\
\hline \multirow[t]{3}{*}{ VII } & Botswana, South Africa & $1987-1998$ & $3(3)$ & B V W V V N A A A A A A F & $425-437$ \\
\hline & & & & B V W V V V V B N A A A B A G & \\
\hline & & & & B V W V V N A A A A A A A F & \\
\hline
\end{tabular}




\begin{tabular}{|l|l|l|l|l|l|}
\hline $\begin{array}{l}\boldsymbol{p} 72 \\
\text { genotype }\end{array}$ & $\begin{array}{l}\text { Southern African countries (other } \\
\text { African countries }\end{array}$ & $\begin{array}{l}\text { Field } \\
\text { presence }\end{array}$ & $\begin{array}{l}\text { No. of } \\
\text { viruses } \\
\text { (no. of } \\
\text { CVR } \\
\text { variants) }\end{array}$ & $\begin{array}{l}\text { Southern African CVR sequence } \\
\text { variants }^{\mathbf{b}}\end{array}$ & $\begin{array}{l}\text { CVR-PCR } \\
\text { product size } \\
\text { range }\end{array}$ \\
\hline VIII & $\begin{array}{l}\text { Mozambique (Malawi, Zambia, } \\
\text { Zimbabwe) }\end{array}$ & 1998 & $\begin{array}{l}\text { A V S V S V S O V N A V N O V V N } \\
\text { V O V O O V }\end{array}$ & 533 \\
\hline XVII & Zimbabwe & 1992 & $1(1)$ & B N A B T D B N CVDT N A F \\
\hline XVIII & Namibia & 1995 & $1(1)$ & $\begin{array}{l}\text { B N A A A A B N A B N B T B N B N } \\
\text { A A A F }\end{array}$ & 509 \\
\hline XIX & South Africa & $1987-1996$ & $5(1)$ & B V W V W V V N A A B A G \\
\hline XX & South Africa & $1973-1995$ & $3(2)$ & B V W B N N A B N B A A G & 413 \\
\hline & & & $1985-1996$ & $2(1)$ & B V W N A A V T A A \\
\hline XXI & South Africa & South Africa & 1992 & B V W V W V V N A A B A G \\
\hline XXII & A A F N N A B N B A A A B N B & 413 \\
\hline
\end{tabular}

${ }^{a}$ African countries outside the southern African region, shown in previous studies (Bastos et al., 2003, Bastos et al., 2004 and Lubisi et al., 2005) to contain the $p 72$ genotype.

${ }^{\mathrm{b}}$ Tetrameric repeat codes are as follows: $\mathrm{A}=\mathrm{CAST} / \mathrm{CVST} / \mathrm{CTST}, \mathrm{B}=\mathrm{CADT} / \mathrm{CTDT}, \mathrm{D}=\mathrm{CASM}, \mathrm{F}=\mathrm{CANT}, \mathrm{G}=\mathrm{CTNT}$, $\mathrm{M}=\mathrm{NEDT}, \mathrm{N}=\mathrm{NVDT} / \mathrm{NVGT} / \mathrm{NVNT}, \mathrm{O}=\mathrm{NANI} / \mathrm{NADI} / \mathrm{NASI}, \mathrm{S}=\mathrm{SAST}, \mathrm{T}=\mathrm{NVNT}, \mathrm{V}=\mathrm{NAST} / \mathrm{NADT} / \mathrm{NANT}$, $\mathrm{W}=\mathrm{SADT} / \mathrm{SVDT}$.

${ }^{\mathrm{c}}$ Product sizes determined empirically from sequencing. 
The results revealed the involvement of a genotype I (ESACWA) virus in the 1989 outbreaks occurring in Windhoek, Namibia. This outbreak was distinct from that occurring 6 years later, again in Windhoek, which was caused by a virus belonging to genotype XVIII. This localised genotype-richness was also observed for ASF viruses from Ellisras and Potgietersrus in South Africa. Each of these areas had small, localised outbreaks due to at least two different genotypes, namely genotypes III (1993 and 1995) and XIX (1987) from Ellisras and genotypes VII (1998) and XIX (1987) from Potgietersrus.

\subsection{Intra-genotypic resolution of homogeneous $p 72$ southern African genotypes}

In order to assess variability within southern African $p 72$ genotypes, the CVR region of 23 viruses was amplified and sequenced in this study. This dataset was supplemented with data from eight additional Mozambican viruses characterised previously (Bastos et al., 2004; Table 1). The results (Table 2) indicate a high degree of variation in amplicon length for viruses from southern Africa, with product sizes ranging from $377 \mathrm{bp}$ (genotypes II and XXI) to $533 \mathrm{bp}$ (genotype VIII), corresponding to 10 and 23 tetrameric repeats, respectively. Within-genotype variation was difficult to assess as 5 of the 14 southern African $p 72$ genotypes were represented by a single virus each, and a further three genotypes by just two viruses. For the better represented genotypes that comprised viruses indistinguishable from each other by $p 72$ gene sequencing alone, such as genotype VII, three different CVR sequence variants were recovered, one from each of the three outbreak strains, and representative of three genetically distinct viruses of either Botswanan or South African origin. In contrast, the five genotype XIX viruses that were identical to each other across the C-terminal end of the $p 72$ gene also had identical CVR sequences, despite being sampled over a 9-year period, indicative of a prolonged field presence in South Africa. Additional epidemiological links confirmed by CVR sequencing included the recovery of identical viruses from the 1993 and 1995 genotype III outbreaks (RSA/5/95 and SPEC/257) in Ellisras. Similarly, the viruses that caused outbreaks in 1973 and 1975 (24823 and Lillie) had identical CVR sequences despite being sampled 2 years apart, but were distinct from the third genotype XX virus (RSA/1/95) recovered from an outbreak in 1995. 
The CVR of the $9 R L$ ORF consisted of repeated amino acid tetramers that varied in number and type, with $\mathrm{CA}(\mathrm{S} / \mathrm{D} / \mathrm{N}) \mathrm{T}$ and $\mathrm{NA}(\mathrm{S} / \mathrm{D} / \mathrm{N}) \mathrm{T}$ being the most common (Table 2). Three tetrameric repeats CTNT (genotypes III, IV, VII, XIX, XX and XXII), SADT (genotypes XXII, XIX and XXI) and SVDT (genotype XX) were identified in this study and have not been previously described (Irusta et al., 1996, Bastos et al., 2004 and Nix et al., 2006). A fourth tetrameric repeat that also appears to be unique to viruses of southern African origin is NEDT (genotypes V and VI), which was previously characterised in a study focussing on viruses of Mozambican origin (Bastos et al., 2004).

\section{Discussion}

The combined $p 72$-CVR sequencing approach first advocated for molecular epidemiological studies based on results obtained from a country-specific study (Bastos et al., 2004) was used here to investigate the relationships of southern African outbreak strains. Phylogenetic analysis of the C-terminal end of the $p 72$ gene revealed high levels of regional heterogeneity, with 14 genotypes being recovered of which 6 were novel and 12 appeared to be confined to the southern African region. These results indicate that $p 72$ genotype-richness for East and southern Africa is comparable, with 13 genotypes being identified from 8 East African countries (Lubisi et al., 2005) and 14 from 5 southern African countries in this study. In contrast, West Africa is genotype-poor with only one genotype (genotype I) being recorded thus far in the numerous ASF-affected countries along the West African seaboard (Bastos et al., 2003, Lubisi et al., 2005 and Nix et al., 2006). Thirteen of the 14 southern African genotypes clustered within evolutionary lineage I which has the widest geographical distribution, mainly due to the incorporation of genotype I (or the ESACWA genotype) which comprises viruses of European, South American, Caribbean and West African origin (Bastos et al., 2003). In contrast lineages II and III have a more restricted distribution, with each being confined to four East African countries (Bastos et al., 2003 and Lubisi et al., 2005), namely Zambia, Mozambique, Malawi and Tanzania (for lineage II), and Tanzania, Kenya, Burundi and Uganda (for lineage III).

Sequencing of CVR-PCR products proved useful for resolving relationships within each of the southern African $p 72$ genotypes that were genetically homogeneous and provided 
new epidemiological insights. The outbreaks occurring in South Africa in 1995 and 1996 and believed to represent two unrelated epizootics, were in fact shown to be due to four genotypically unrelated viruses (genotypes III and XX in 1995 and genotypes XIX and XXI in 1996) and therefore of four unrelated origins. These results contrast markedly with the recovery of identical genotype XIX viruses from the temporally unrelated (1987, 1992 and 1996) outbreaks in South Africa, indicating a prolonged field presence for this virus. As the C-terminal end of the $p 72$ gene of viruses from domestic pig cycles in West and East Africa (genotypes I and VIII, respectively) are genetically homogeneous (Bastos et al., 2003 and Lubisi et al., 2005), it raises the possibility the genotype XIX homogeneity observed here may imply the existence of a pig-restricted cycle in South Africa, in addition to the recognised sylvatic cycle, which would have serious implications for disease control (Penrith et al., in press). However, this requires further investigation as only five genotype XIX have been characterised thus far whilst in excess of 40 viruses each have been sequenced for genotypes I and VIII (Bastos et al., 2003 and Lubisi et al., 2005).

CVR size variability for the 14 southern African $p 72$ genotypes ranged from 377 bp to $533 \mathrm{bp}$ with nine PCR size-discrete classes being identified. This is relatively low when compared with the 16 size-discrete classes recovered from viruses representative of a single genotype (genotype I) where the CVR PCR product sizes ranged from $360 \mathrm{bp}$ to 686 bp (Phologane et al., 2005 and Nix et al., 2006), and brings into question the usefulness of the CVR for molecular epidemiological studies in the southern African context. However, despite the relatively lower level of size variability, the 9 size-discrete CVR products identified here produced 13 sequence-discrete classes, and provided some within-genotype resolution for viruses that were homogeneous across $p 72$ and that contained three or more virus representatives. The apparent restricted usefulness of CVR in southern Africa, may therefore be an artefact of sample size, but this will only be known once more viruses of the under-represented genotypes (XXVII, XXVIII, XXI and XXII) become available for characterisation.

This study confirms previous cautionary notes on the use of CVR for inferring acrossgenotype viral relationships (Bastos et al., 2004) and has identified the need for plasticity when delineating $p 72$ genotypes. With respect to the latter, it appears that it is possible 
that two viruses belonging to a single monophyletic lineage or genotype can exceed the $0.96 \%$ cut-off advocated for $p 72$ genotype delineation in combination with bootstrap support (Bastos et al., 2003). This is particularly so for viruses from some East African genotypes (Lubisi, 2005). In contrast, viruses of some of the southern African genotypes identified in this study were shown to have sequence identity values that are below $0.96 \%$ when compared with viruses from other genotypes, and yet clearly belong to unrelated lineages. This stresses the importance of using the $0.96 \%$ cut-off as a guide only, rather than an absolute value when defining monophyletic groups of ASF viruses at the genotype level. With respect to CVR, although it has previously been shown that the CVR-PCR sequencing approach is useful for differentiating genotype I viruses (Irusta et al., 1996, Phologane et al., 2005 and Nix et al., 2006) and those recovered from outbreaks in Mozambique (Bastos et al., 2004), its use should be limited to within-genotype comparisons. This is due to the hypervariable nature of this genome region, which not only introduces bias when aligning the tetrameric repeat regions (Bastos et al., 2004) but which, like microsatellite data, is inherently homoplasic and therefore only useful for clarifying relationships of closely related taxa.

The results obtained in this study expand on and confirm the usefulness of a combined p72-CVR characterisation approach for investigating the relatedness of ASF epizootics (Bastos et al., 2004 and Nix et al., 2006). Valuable insights into the heterogeneity and relatedness of ASF viruses recovered from outbreaks in southern Africa have been obtained and should be expanded to address the role of all sylvatic hosts. This will lead to a better understanding of the origin and spread of the disease in this region.

\section{References}

Anderson et al., 1998 E.C. Anderson, G.H. Hutchings, N. Mukarati and P.J. Wilkinson, African swine fever virus infection of the bushpig (Potamochoerus porcus) and its significance in the epidemiology of the disease, Vet. Microbiol. 62 (1998), pp. 1-15. Bastos et al., 2003 A.D.S. Bastos, M.-L. Penrith, C. Crucière, J.L. Edrich, G. Hutchings, F. Roger, E. Couacy-Hymann and G.R. Thomson, Genotyping field strains of African swine fever virus by partial $p 72$ gene characterization, Arch. Virol. 148 (2003), pp. 693706. 
Bastos et al., 2004 A.D. Bastos, M.L. Penrith, F. Macome, F. Pinto and G.R. Thomson, Co-circulation of two genetically distinct viruses in an outbreak of African swine fever in Mozambique: no evidence for individual co-infection, Vet. Microbiol. 103 (2004), pp. 169-182.

Blasco et al., 1989 R. Blasco, M. Aguero, J.M. Almendral and E. Viñuela, Variable and constant regions in African swine fever virus DNA, Virology 168 (1989), pp. 330-338. Dixon and Wilkinson, 1988 L.K. Dixon and P.J. Wilkinson, Genetic diversity of African swine fever isolates from soft ticks (Ornithodoros moubata) inhabiting warthog burrows in Zambia, J. Gen. Virol. 69 (1988), pp. 2981-2993.

Dixon et al., 2000 L.K. Dixon, J.V. Costa, J.M. Escribano, D.L. Rock, E. Viñuela and P.J. Wilkinson, Family Asfarviridae. In: M.H.V. Van Regenmortel, C.M. Fauquet, D.H.L. Bishop, E.B. Carestens, M.K. Estes, S.M. Lemon, J. Maniloff, M.A. Mayo, D.J. McGeoch, C.R. Pringle, R.B.F.A. Wickner, C.M. Murphy, D.H.L. Fauquet, S.A. Bishop, A.W. Ghabrial, G.P. Jarvis and M.D. Martelli, Editors, Virus Taxonomy: Seventh Report of the International Committee on Taxonomy of Viruses, Summers Academic Press, San Diego (2000), pp. 159-165.

Huelsenbeck and Ronquist, 2003 J.P. Huelsenbeck and F. Ronquist, MrBayes: A Program for the Bayesian Inference of Phylogeny, 3.0, Rochester, New York (2003). Irusta et al., 1996 P.M. Irusta, M.V. Borca, G.F. Kutish, Z. Lu, E. Caler, C. Carrillo and D.L. Rock, Amino acid tandem repeats within a late viral gene define the central variable region of African swine fever virus, Virology 220 (1996), pp. 20-27.

Kumar et al., 2004 S. Kumar, K. Tamura and M. Nei, MEGA3: Integrated software for Molecular Evolutionary Genetics Analysis and sequence alignment, Brief. Bioinform. 5 (2004), pp. 150-163.

Lubisi, 2005 Lubisi, B.A., 2005. Molecular epidemiology of African swine fever in East Africa. M.Sc. Dissertation. University of Pretoria, pp. 27-83.

Lubisi et al., 2005 B.A. Lubisi, A.D. Bastos, R.M. Dwarka and W. Vosloo, Molecular epidemiology of African swine fever in East Africa, Arch. Virol. 150 (2005), pp. 2439 2452. 
Malmquist and Hay, 1960 W.A. Malmquist and D. Hay, Haemadsorption and cytopathic effect produced by African swine fever virus in swine bone marrow and buffy coat cultures, Am. J. Vet. Res. 21 (1960), pp. 104-108.

Montgomery, 1921 R.E. Montgomery, On a form of swine fever occurring in British East Africa (Kenya Colony), J. Comp. Pathol. 34 (1921), pp. 159-191.

Nix et al., 2006 R.J. Nix, C. Gallardo, G. Hutchings, E. Blanco and L.K. Dixon, Molecular epidemiology of African swine fever virus studied by analysis of four variable genome regions, Arch. Virol. 151 (2006), pp. 2475-2494.

Penrith, 2005 M.-L. Penrith, Control of African swine fever for smallholder pig producers in sub-Saharan Africa, Proceedings of the Vet Africa Congress Johannesburg, South Africa, 30th July-1st August 2005 (2005), pp. 63-70.

Penrith et al., 2004 M.-L. Penrith, G.R. Thomson and A.D.S. Bastos, African swine fever. In: J.A.W. Coetzer and R.C. Tustin, Editors, Infectious Diseases of Livestock with Special Reference to Southern Africa (2nd ed.), Oxford University Press, Cape Town, London, New York (2004), pp. 1087-1119.

Penrith et al., in press Penrith, M.L., Lopes Pereira, C., Lopes da Silva, M.M.R., Quembo, C., Nhamusso, A., Banze, J., in press. African swine fever in Mozambique: review, risk factors and considerations for control. Onderstepoort J. Vet. Res. Phologane et al., 2005 S.B. Phologane, A.D.S. Bastos and M.L. Penrith, Intra- and intergenotypic size variation in the central variable region of the 9RL open reading frame of diverse African swine fever viruses, Virus Genes 31 (2005), pp. 357-360.

Pini and Hurter, 1975 A. Pini and L.R. Hurter, African swine fever: an epizootiological review with special reference to the South African situation, J. S. Afr. Vet. Ass. 46 (1975), pp. 227-232.

Posada and Crandall, 1998 D. Posada and K.A. Crandall, ModelTest: testing the model of DNA substitution, Bioinformatics 14 (1998), pp. 817-818.

Steyn, 1932 D.G. Steyn, East African virus disease in pigs, Proceedings of the 18th Report of the Director of Veterinary Services and Animal Industry, vol. 1 Union of South Africa (1932), pp. 99-109.

Swofford, 2003 D.L. Swofford, PAUP*. Phylogenetic Analysis Using Parsimony (*and Other Methods). Version 4.10 MA, Sinauer Associates, Inc., USA (2003). 
Thomson et al., 1980 G.R. Thomson, G. Gainaru and A.F. van Dellen, Experimental infection of warthog (Phacochoerus aethiopicus) with African swine fever virus, Onderstepoort J. Vet. Res. 47 (1980), pp. 19-22.

Vosloo et al., 2006 W. Vosloo, A.D.S. Bastos and C.I. Boshoff, Retrospective genetic analysis of SAT-1 type foot-and-mouth disease outbreaks in southern Africa, Arch. Virol. 151 (2006), pp. 285-298.

Wesley and Tuthill, 1984 R.D. Wesley and A.E. Tuthill, Genome relatedness among African swine fever virus field isolates by restriction endonuclease analysis, Prev. Vet. Med. 2 (1984), pp. 53-62.

Yu et al., 1996 M. Yu, C.J. Morrissy and H.A. Westbury, Strong sequence conservation of African swine fever virus $\mathrm{p} 72$ protein provides the molecular basis for its antigenic stability, Arch. Virol. 141 (1996), pp. 1795-1802.

Corresponding author at: Mammal Research Institute, Department of Zoology and Entomology, University of Pretoria, Pretoria 0002, South Africa. Tel.: +27 12420 4612; fax: +27123625242 . 